\title{
Recovering Differential Operators on the Half-Line with Indefinite Discontinuous Weights
}

\author{
Vjacheslav Yurko
}

\begin{abstract}
Non-self-adjoint second-order differential operators on the half-line with indefinite discontinuous weights are studied. Properties of spectral characteristics are established and inverse problems of recovering operators from their spectral characteristics are investigated. For these class of nonlinear inverse problems algorithms for constructing the global solutions are developed, and uniqueness theorems are proved.
\end{abstract}

\section{Introduction}

We consider the following differential equation

$$
-y^{\prime \prime}(x)+q(x) y(x)=\lambda r(x) y(x), \quad x>0 .
$$

Here $\lambda$ is the spectral parameter, $r(x)=-\beta^{2}$ for $x<a, r(x)=\alpha^{2}$ for $x>a$, and $\alpha>0$, $\beta>0, a \in(0, \pi)$. The function $q(x)$ is complex-valued, and $(1+x) q(x) \in L(0, \infty)$. Denote by $\mathcal{L}$ the non-self-adjoint boundary value problem for Eq. (1.1) with the boundary condition

$$
U(y):=y^{\prime}(0)-h y(0)=0,
$$

and with the jump conditions

$$
y(a+0)=a_{1} y(a-0), y^{\prime}(a+0)=y^{\prime}(a-0) / a_{1}+a_{2} y(a-0), a_{1} \neq 0,
$$

here $h, a_{1}, a_{2}$ are complex numbers, and $a_{ \pm}:=a_{1} \pm i \beta /\left(\alpha a_{1}\right) \neq 0$. The function $r(x)$ changes sign in the interior point $x=a$ which is called the turning point. In this paper we establish properties of spectral characteristics of $\mathcal{L}$, and study inverse problems of recovering $\mathcal{L}$ from its spectral characteristics. For definiteness, let $\operatorname{Re} a_{1}>0$ or $\operatorname{Re} a_{1}=0, \operatorname{Im} a_{1}>0$.

2010 Mathematics Subject Classification. 34A55, 34B24, 47E05.

Key words and phrases. differential operators; indefinite discontinuous weights; inverse spectral problems; method of spectral mappings..

Corresponding author: Vjacheslav Yurko. 
Differential equations with turning points and indefinite weights arise in various areas of mathematics as well as in applications (see $[1,2,3]$ for details). In particular, they appear in elasticity, optics, geophysics, electronics and other branches of natural sciences and engineering. Moreover, a wide class of differential equations with Bessel-type singularities and their perturbations can be reduced to differential equations with turning points and indefinite weights. It is also known that inverse spectral problems play an important role for solving nonlinear integrable evolution equations ( $\mathrm{KdV}$ equation and others). Inverse problems for equations with turning points and indefinite weights help to study the blow-up behavior of solutions for such nonlinear equations.

Inverse spectral problems for the classical Sturm-Liouville equation $-y^{\prime \prime}+q(x) y=\lambda y$, have been studied fairly completely (see [4] and the references therein). The presence of turning points and indefinite weights in the differential equation produces essential qualitative modifications in the investigations of inverse problems. Inverse problems for the Sturm-Liouville equation on the half-line with a smooth indefinite weight were studied in [5]. Inverse problems for the boundary value problem (1.1)-(1.3) have not been studied yet. For solving these class of inverse problems we develop ideas of the method of spectral mappings [6]. Using this approach we obtain algorithms for constructing the global solutions of these nonlinear inverse problems, and prove the corresponding uniqueness theorems.

\section{Auxiliary propositions}

Let $\varphi(x, \lambda)$ and $S(x, \lambda)$ be solutions of Eq. (1.1) satisfying (1.3) and the initial conditions $\varphi(0, \lambda)=$ $S^{\prime}(0, \lambda)=1, \varphi^{\prime}(0, \lambda)=h, S(0, \lambda)=0$. For each fixed $x$, the functions $\varphi^{(\nu)}(x, \lambda)$ and $S^{(\nu)}(x, \lambda), \nu=0,1$, are entire in $\lambda$ of order $1 / 2$, and

$$
\langle\varphi(x, \lambda), S(x, \lambda)\rangle \equiv 1
$$

where $\langle y(x), z(x)\rangle:=y(x) z^{\prime}(x)-y^{\prime}(x) z(x)$ is the Wronskian of $y$ and $z$.

Let $\lambda=\rho^{2}, \operatorname{Im} \rho \geq 0, \rho=\sigma+i \tau$, and let $\Pi_{+}$be the $\lambda$-plane with the two-sided cut $\Pi_{0}$ along the arc $B_{+}:=\{\lambda: \quad \lambda \geq 0\}$. Put $\Pi:=\overline{\Pi_{+}} \backslash\{0\}$. The sets $\Pi_{+}, \Pi_{0}$ and $\Pi$ corresponds

to the sets $\Lambda_{+}:=\{\rho: \operatorname{Im} \rho>0\}, \Lambda_{0}:=\{\rho: \operatorname{Im} \rho=0\}, \Lambda:=\{\rho: \operatorname{Im} \rho \geq 0, \rho \neq 0\}$, respectively.

Let $E(x, \rho), x \geq 0, \operatorname{Im} \rho \geq 0$, be the solution of Eq. (1.1) satisfying (1.3) and the condition $E(x, \rho) \exp (-i \alpha \rho x) \rightarrow 1$ as $x \rightarrow \infty$. 
Lemma 2.1. For each $x$, the functions $E^{(\nu)}(x, \rho), \nu=0,1$, are regular for $\rho \in \Lambda_{+}$, are continuous for $\operatorname{Im} \rho \geq 0$, and are continuously differentiable for $\rho \in \Lambda$. For $|\rho| \rightarrow \infty, \operatorname{Im} \rho \geq 0, \nu=0,1$, the following asymptotical formulas hold:

$$
\begin{gathered}
E^{(\nu)}(x, \rho)=(i \alpha \rho)^{\nu} \exp (i \alpha \rho x)[1], \quad x>a, \\
E^{(\nu)}(x, \rho)=\frac{(i \alpha)(\beta \rho)^{\nu}}{2 \beta} \exp (i \alpha \rho a)\left(a_{-} \exp (\beta \rho(x-a))[1]+\right. \\
\left.(-1)^{\nu} a_{+} \exp (-\beta \rho(x-a))[1]\right), x<a,
\end{gathered}
$$

where $[1]=1+O\left(\rho^{-1}\right)$. Moreover, for real $\rho \neq 0$,

$$
\langle E(x, \rho), E(x,-\rho)\rangle \equiv-2 i \rho .
$$

Proof. Let $e(x, \rho)$ be the Jost solution for Eq. (1.1) (see [4, Ch. 2]). Then $E(x, \rho) \equiv e(x, \rho)$ for $x>a$, hence (2.2) is valid. Consider the sectors $S_{j}:=\{\rho: \arg \rho \in[j \pi / 2,(j+1) \pi / 2]\}$, $S_{\delta}^{0}:=\{\rho: \arg \rho \in[\pi / 2-\delta, \pi / 2+\delta]\}, S_{j, \delta}:=S_{j} \backslash S_{\delta}^{0}, j=0,1,0<\delta<\pi / 4$. It is known (see, e.g. [4, Ch.1]) that in each sector $S_{j}$ there exists a fundamental system of solutions of Eq. (1.1) $\left\{y_{k}(x, \rho)\right\}_{k=1,2}$ for $x \in[0, a]$ such that

$$
y_{k}^{(\nu)}(x, \rho)=\left((-1)^{k-1} \beta \rho\right)^{\nu} \exp \left((-1)^{k-1} \beta \rho x\right)[1],|\rho| \rightarrow \infty, \rho \in S_{j}, x \in[0, a], \nu=0,1 .
$$

Hence we can write

$$
E(x, \rho)=A_{1}(\rho) y_{1}(x, \rho)+A_{2}(\rho) y_{2}(x, \rho), x \in[0, a]
$$

where $A_{k}(\rho)$ do not depend on $x$. Using (1.3) and (2.2) we obtain for $|\rho| \rightarrow \infty, \rho \in S_{j}$ :

$$
A_{1}(\rho)=\frac{i \alpha a_{-}}{2 \beta} \exp (i \alpha \rho a) \exp (-\beta \rho a)[1], A_{2}(\rho)=-\frac{i \alpha a_{+}}{2 \beta} \exp (i \alpha \rho a) \exp (\beta \rho a)[1] .
$$

Substituting these relations into (2.5) we arrive at (2.3). The other assertions of the lemma follow from the properties of $e(x, \rho)$. Lemma 1 is proved.

Analogously one can prove that for $|\rho| \rightarrow \infty, \operatorname{Im} \rho \geq 0, \nu=0,1$, the following asymptotical formulas hold:

$$
\begin{gathered}
\varphi^{(\nu)}(x, \lambda)=\left((\beta \rho)^{\nu} \exp (\beta \rho x)[1]+(-\beta \rho)^{\nu} \exp (-\beta \rho x)[1]\right) / 2, x \in[0, a-0] \\
\varphi^{(\nu)}(x, \lambda)=\left(\left(a_{-} \exp (\beta \rho a)[1]+a_{+} \exp (-\beta \rho a)[1]\right)(i \alpha \rho)^{\nu} \exp (i \alpha \rho(x-a))[1]+\right. \\
\left.\left(a_{+} \exp (\beta \rho a)[1]+a_{-} \exp (-\beta \rho a)[1]\right)(-i \alpha \rho)^{\nu} \exp (-i \alpha \rho(x-a))[1]\right) / 4,
\end{gathered}
$$


$x \in[a+0, \infty)$. Consider the function

$$
\Delta(\rho):=E^{\prime}(0, \rho)-h E(0, \rho)
$$

Clearly, $\Delta(\rho)$ is regular for $\rho \in \Lambda_{+}$, is continuous for $\operatorname{Im} \rho \geq 0$, and is continuously differentiable for $\rho \in \Lambda$. It follows from (2.3) and (2.8) that

$$
\Delta(\rho)=(i \alpha \rho) \exp (i \alpha \rho a)\left(a_{+} \exp (\beta \rho a)[1]+a_{-} \exp (-\beta \rho a)[1]\right) / 2,
$$

$|\rho| \rightarrow \infty, \operatorname{Im} \rho \geq 0$. Analogously we obtain for $|\rho| \rightarrow \infty, \operatorname{Im} \rho \geq 0$ :

$$
\begin{gathered}
E(0, \rho)=(i \alpha) \exp (i \alpha \rho a)\left(a_{-} \exp (-\beta \rho a)[1]-a_{+} \exp (\beta \rho a)[1]\right) /(2 \beta) \\
\Delta_{1}(\rho)=(i \alpha \rho) \exp (i \alpha \rho a)\left((i \alpha+\beta) a_{+} \exp (\beta \rho a)[1]+(i \alpha-\beta) a_{-} \exp (-\beta \rho a)[1]\right) / 4
\end{gathered}
$$

here $\Delta_{1}(\rho)=\frac{d}{d \lambda} \Delta(\rho)=\frac{1}{2 \rho} \frac{d}{d \rho} \Delta(\rho)$. It follows from (2.9) and (2.11) that for large $|\rho|$, the function $\Delta(\rho)$ has simple zeros

$$
\rho_{k}=i((k \pi) /(\beta a)+\theta)+O\left(k^{-1}\right), k \rightarrow \infty, \quad \theta=(2 i \beta a)^{-1} \ln \left(-a_{-} / a_{+}\right) .
$$

Moreover,

$$
|\Delta(\rho)| \geq C|\rho| \exp (\beta|\sigma| a) \exp (\alpha \tau a), \quad \rho \in G_{\delta},
$$

where $G_{\delta}:=\left\{\rho: \operatorname{Im} \rho \geq 0,\left|\rho-\rho_{k}\right| \geq \delta\right\}$.

\section{Inverse problem from the Weyl function}

Consider the function

$$
\Phi(x, \lambda):=E(x, \rho) / \Delta(\rho)
$$

and put $M(\lambda):=\Phi(0, \lambda)$. The function $M(\lambda)$ will be called the Weyl function. By virtue of (3.1),

$$
M(\lambda)=E(0, \rho) / \Delta(\rho)
$$

It is easy to check that

$$
\Phi(x, \lambda)=S(x, \lambda)+M(\lambda) \varphi(x, \lambda) .
$$


According to (2.1) and (3.3), we have

$$
\langle\varphi(x, \lambda), \Phi(x, \lambda)\rangle \equiv 1
$$

Denote $B:=\left\{\lambda=\rho^{2}: \rho \in \Lambda, \Delta(\rho)=0\right\}, B^{\prime}:=\left\{\lambda=\rho^{2}: \rho \in \Lambda_{+}, \Delta(\rho)=0\right\}$, $B^{\prime \prime}:=\left\{\lambda=\rho^{2}: \rho \in \Lambda_{0}, \rho \neq 0, \Delta(\rho)=0\right\}$. Then $B=B^{\prime} \cup B^{\prime \prime}$. Clearly, $B^{\prime}$ is a countable set, and $B^{\prime \prime}$ is a bounded set. The function $M(\lambda)$ is regular in $\Pi_{+} \backslash B^{\prime}$, and continuously differentiable in $\Pi \backslash B$. The set of singularities of $M(\lambda)$ (which is called the spectrum of $\mathcal{L}$ and is denoted by $s(\mathcal{L})$ ) coincides with the set $B_{0}:=B_{+} \cup B$. The values of $\lambda$, for which Eq. (1.1) has non-trivial solutions satisfying (1.2), (1.3) and the condition $y(\infty)=0$ are called eigenvalues of $\mathcal{L}$. By virtue of (2.9), (2.10) and (3.2), we get

$$
M(\lambda)=(-1)^{j+1}(\beta \rho)^{-1}[1],|\rho| \rightarrow \infty, \rho \in S_{j, \delta}
$$

Theorem 3.1. There are no eigenvalues for $\lambda>0$. The set $B^{\prime}$ coincides with the set of non-zero eigenvalues. If $\lambda_{0}=\rho_{0}^{2}>0$ and $\Delta\left(\rho_{0}\right)=0$, then $\Delta\left(-\rho_{0}\right) \neq 0$.

Proof. Let $\lambda_{0}=\rho_{0}^{2}>0$ be an eigenvalue with an eigenfunction $y_{0}(x)$. Then $y_{0}(x)=A_{+} E\left(x, \rho_{0}\right)+$ $A_{-} E\left(x,-\rho_{0}\right)$. As $x \rightarrow \infty$ one has $y_{0}(x) \sim 0, E\left(x, \pm \rho_{0}\right) \sim \exp \left( \pm i \alpha \rho_{0} x\right)$. Hence $A_{+}=A_{-}=$ 0 . If $\lambda_{0}=\rho_{0}^{2}>0$ and $\Delta\left(\rho_{0}\right)=0$, then it follows from (2.8) and (2.4) that $E\left(0, \rho_{0}\right) \Delta\left(-\rho_{0}\right) \neq 0$, and consequently, $\Delta\left(-\rho_{0}\right) \neq 0$.

If $\lambda_{0}=\rho_{0}^{2} \in B^{\prime}$, then $U\left(E\left(x, \rho_{0}\right)\right)=\Delta\left(\rho_{0}\right)=0$, i.e. $E\left(x, \rho_{0}\right)$ is an eigenfunction, and $\lambda_{0}=\rho_{0}^{2}$ is an eigenvalue. Conversely, let $\lambda_{0}=\rho_{0}^{2}, \operatorname{Im} \rho_{0}>0$ be an eigenvalue with an eigenfunction $y_{0}(x)$. Then $d_{0}:=y_{0}(0) \neq 0, y_{0}^{\prime}(0)=d_{0} h$, and consequently, $y_{0}(x) \equiv d_{0} \varphi\left(x, \lambda_{0}\right)$. On the other hand, $y_{0}(x)=d E\left(x, \rho_{0}\right), d \neq 0$. Therefore, $\Delta\left(\rho_{0}\right)=U\left(E\left(x, \rho_{0}\right)\right)=0$, i.e. $\lambda_{0} \in B^{\prime}$.

Without loss of generality we will assume below that $\alpha=1$. In this section we consider the following inverse problem.

Inverse problem 1. Given the Weyl function $M(\lambda)$, construct $\mathcal{L}$.

Let us prove the uniqueness theorem for this inverse problem. For this purpose, together with $\mathcal{L}$ we consider a boundary value problem $\tilde{\mathcal{L}}$ of the same form but with $\tilde{q}(x), \tilde{a}, \tilde{\beta}, \tilde{h}, \tilde{a}_{1}, \tilde{a}_{2}$ instead of $q(x), a, \beta, h, a_{1}, a_{2}$. We agree that if a certain symbol $\chi$ denotes an object related to $\mathcal{L}$, then $\tilde{\chi}$ will denote an analogous object related to $\tilde{\mathcal{L}}$, and $\hat{\chi}:=\chi-\tilde{\chi}$.

Theorem 3.2. If $M(\lambda)=\tilde{M}(\lambda)$, then $\mathcal{L}=\tilde{\mathcal{L}}$, i.e. $q(x)=\tilde{q}(x)$ a.e., $a=\tilde{a}, \beta=\tilde{\beta}, h=\tilde{H}$, $a_{1}=\tilde{a}_{1}, a_{2}=\tilde{a}_{2}$. Thus, the specification of the Weyl function $M(\lambda)$ uniquely determines $\mathcal{L}$. 
Proof. Using (3.5) we calculate

$$
\beta=-\left(\lim _{|\rho| \rightarrow \infty} \rho M(\lambda)\right)^{-1}, \arg \rho \in[0, \pi / 4] .
$$

Since $M(\lambda)=\tilde{M}(\lambda)$, it follows that $\beta=\tilde{\beta}$. Furthermore, $\rho_{k}=\tilde{\rho}_{k}$, and by virtue of (2.12), $a=\tilde{a}$ and $a_{1}=\tilde{a}_{1}$. Consider the functions

$$
\begin{aligned}
& T_{1}(x, \lambda)=\varphi(x, \lambda) \tilde{\Phi}^{\prime}(x, \lambda)-\Phi(x, \lambda) \tilde{\varphi}^{\prime}(x, \lambda), \\
& T_{2}(x, \lambda)=\Phi(x, \lambda) \tilde{\varphi}(x, \lambda)-\varphi(x, \lambda) \tilde{\Phi}(x, \lambda) .
\end{aligned}
$$

Using (3.4) and (3.7) we calculate

$$
\begin{gathered}
\varphi(x, \lambda)=T_{1}(x, \lambda) \tilde{\varphi}(x, \lambda)+T_{2}(x, \lambda) \tilde{\varphi}^{\prime}(x, \lambda), \\
\Phi(x, \lambda)=T_{1}(x, \lambda) \tilde{\Phi}(x, \lambda)+T_{2}(x, \lambda) \tilde{\Phi}^{\prime}(x, \lambda), \\
T_{1}(x, \lambda)-1=\varphi(x, \lambda)\left(\tilde{\Phi}^{\prime}(x, \lambda)-\Phi^{\prime}(x, \lambda)\right)-\Phi(x, \lambda)\left(\tilde{\varphi}^{\prime}(x, \lambda)-\varphi^{\prime}(x, \lambda)\right) .
\end{gathered}
$$

Taking (3.7), (3.9), (2.13)-(3.1), (2.2)-(2.3) and (2.6)-(2.7) into account, we obtain for $\rho \in G_{\delta} \cap \tilde{G}_{\delta}$ :

$$
\left|T_{1}(x, \lambda)-1\right| \leq C /|\rho|, \quad\left|T_{2}(x, \lambda)\right| \leq C /|\rho| .
$$

Since $M(\lambda)=\tilde{M}(\lambda)$, it follows from (3.3) and (3.7) that for each fixed $x$, the functions $T_{k}(x, \lambda)$, $k=1,2$, are entire in $\lambda$ of order $1 / 2$. Together with (3.10) this yields $T_{1}(x, \lambda) \equiv 1, T_{2}(x, \lambda) \equiv 0$. By virtue of (3.8), one has $\varphi(x, \lambda) \equiv \tilde{\varphi}(x, \lambda), \Phi(x, \lambda) \equiv \tilde{\Phi}(x, \lambda)$, and consequently, $\mathcal{L}=\tilde{\mathcal{L}}$. Theorem 3.2 is proved.

\section{Inverse problem from the spectral data}

In this section, for brevity, we confine ourselves to the case of a simple spectrum in the following sense: we will say that $\mathcal{L}$ has a simple spectrum if all zeros of $\Delta(\rho)$ are simple, have no limit points, and $\rho M(\lambda)=O(1)$ as $\rho \rightarrow 0$.

Suppose that the spectrum of $\mathcal{L}$ is simple. Then $B=\left\{\rho_{k}^{2}\right\}_{k \in \omega}$, where $\omega=\omega_{0} \cup \omega^{0}, B^{\prime \prime}=$ $\left\{\rho_{k}^{2}\right\}_{k \in \omega_{0}}, B^{\prime}=\left\{\rho_{k}^{2}\right\}_{k \in \omega^{0}}, \omega_{0}$ is a finite set of indices, $\omega^{0}$ is a countable set of indices $\left(\omega^{0}=\right.$ $\left\{k ; k>k_{0}\right\}$ for some $k_{0}$ ), and (2.12) holds $k \in \omega^{0}$. Let

$$
\begin{gathered}
M_{k}=\frac{E\left(0, \rho_{k}\right)}{\Delta_{1}\left(\rho_{k}\right)} \neq 0, \rho_{k} \in B ; \quad \alpha_{k}=\left\{\begin{array}{l}
M_{k}, \quad \rho_{k} \in B^{\prime}, \\
M_{k} / 2, \quad \rho_{k} \in B^{\prime \prime},
\end{array}\right. \\
V(\lambda):=\frac{1}{2 \pi i}\left(M^{-}(\lambda)-M^{+}(\lambda)\right), \lambda>0 ; \quad M^{ \pm}(\lambda):=\lim _{z \rightarrow 0, \operatorname{Re} z>0} M(\lambda \pm i z) .
\end{gathered}
$$


Then

$$
\lim _{\lambda \rightarrow \lambda_{k}, \lambda \in \Pi}\left(\lambda-\lambda_{k}\right) M(\lambda)=M_{k}
$$

Using (2.10)-(2.12) and (4.1) we calculate

$$
\alpha_{k}=-2[1] /\left(a \beta^{2}\right), \quad k \rightarrow \infty .
$$

According to (3.2) and (4.2), one has

$$
V(\lambda)=\frac{1}{2 \pi i}\left(\frac{E(0,-\rho)}{\Delta(-\rho)}-\frac{E(0, \rho)}{\Delta(\rho)}\right), \quad \rho>0 .
$$

Taking (2.4) into account, we infer

$$
V(\lambda)=\frac{\rho}{\pi \Delta(\rho) \Delta(-\rho)}, \quad \rho>0 .
$$

The data $S:=\left(\{V(\lambda)\}_{\lambda>0},\left\{\lambda_{k}, \alpha_{k}\right\}_{k \in \omega}\right)$ are called the spectral data of $\mathcal{L}$. It follows from the above arguments that the following properties hold:

1) $\rho_{k} \neq \rho_{s}$ for $k \neq s$; if $\rho_{k} \in B^{\prime \prime}$, then $-\rho_{k} \notin B^{\prime \prime}$.

2) $\alpha_{k} \neq 0$, and (2.12) and (4.4) hold.

3) $\rho V(\lambda)=O(1)$ as $\lambda \rightarrow 0 ; \rho V(\lambda)=O(\exp (-2 \beta \rho a))$ as $\rho>0, \rho \rightarrow \infty$.

4) $V(\lambda)$ is continuously differentiable for $B_{+} \backslash B^{\prime \prime}$.

5) For $\lambda_{k} \in B^{\prime \prime}$ there exist finite limits $V_{k}:=\lim _{\lambda \rightarrow \lambda_{k}}\left(\lambda-\lambda_{k}\right) V(\lambda)$, and

$$
V_{k}=i \alpha_{k} \pi^{-1} \operatorname{sign} \rho_{k}
$$

Relation (4.6) is a connection between $V(\lambda)$, which describes the continuous spectrum, and $\left\{\lambda_{k}, \alpha_{k}\right\}, \lambda_{k} \in B^{\prime \prime}$, related to the discrete spectrum. Let $\Omega_{\delta}:=B_{+} \backslash\left(\cup_{\lambda_{k} \in B^{\prime \prime}}\left[\lambda_{k}-\delta, \lambda_{k}+\delta\right]\right)$.

Theorem 4.1. The specification of the spectral data $S$ uniquely determines the Weyl function $M(\lambda)$ via the formula:

$$
M(\lambda)=\sum_{\lambda_{k} \in B} \frac{\alpha_{k}}{\lambda-\lambda_{k}}+\int_{0}^{\infty} \frac{V(\mu)}{\lambda-\mu} d \mu, \quad \lambda \notin s(\mathcal{L}),
$$

where the integral is understood in the principal value sense: $\int_{0}^{\infty}:=\lim _{\delta \rightarrow 0} \int_{\Omega_{\delta}}$.

Proof. Take $r_{N}=\left((N+\chi) \pi(\beta a)^{-1}\right)^{2}>0$ such that the circles $R_{N}:=\left\{\lambda:|\lambda|=r_{N}\right\}$ lie in $G_{\delta}$ for sufficiently small $\delta>0$, and consider the contour integral

$$
I_{N}(\lambda):=\frac{1}{2 \pi i} \int_{R_{N}} \frac{M(\mu)}{\lambda-\mu} d \mu, \lambda \in \operatorname{int} R_{N}
$$


with counterclockwise circuit. It follows from (3.2), (2.10) and (2.13) that $|M(\lambda)| \leq C|\rho|^{-1}, \lambda \in$ $G_{\delta}$, and consequently, $\lim _{N \rightarrow \infty} I_{N}(\lambda)=0$. For each $\lambda_{k} \in B^{\prime \prime}$ on the upper (lower) side of the cut $\Pi_{0}$ we take a semicircle $\kappa_{\delta}\left(\lambda_{k}\right):=\left\{\lambda:\left|\lambda-\lambda_{k}\right|=\delta, \operatorname{Im} \lambda>0(\operatorname{Im} \lambda<0)\right\}$, and choose $\delta>0$ such that the sets int $\kappa_{\delta}\left(\lambda_{k}\right)$ do not intersect each other, and do not contain points of $B^{\prime}$. Let $\Pi_{\delta}$ be the two-sided cut $\Pi_{0}$ without the $\delta$ - neighbourhoods of the points of $B^{\prime \prime}$, and let $\Gamma_{\delta}:=$ $\Pi_{\delta} \cup\left(\cup_{\lambda_{k} \in B^{\prime \prime}} \kappa_{\delta}\left(\lambda_{k}\right)\right)$ be the contour with counterclockwise circuit. Denote $\Gamma_{\delta, N}:=\Gamma_{\delta} \cap$ int $R_{N}$. Contracting the contour $R_{N}$ in (4.8) to the real axis and using the residue theorem, we get

$$
I_{N}(\lambda)=\frac{1}{2 \pi i} \int_{\Gamma_{\delta, N}} \frac{M(\mu)}{\lambda-\mu} d \mu+\sum_{\lambda_{k} \in B^{\prime},\left|\lambda_{k}\right|<r_{N}} \frac{\alpha_{k}}{\lambda-\lambda_{k}}-M(\lambda) .
$$

This yields as $N \rightarrow \infty$ :

$$
M(\lambda)=\sum_{\lambda_{k} \in B^{\prime}} \frac{\alpha_{k}}{\lambda-\lambda_{k}}+\frac{1}{2 \pi i} \int_{\Gamma_{\delta}} \frac{M(\mu)}{\lambda-\mu} d \mu .
$$

Using (4.1) and (4.3) we obtain for each $\lambda_{k} \in B^{\prime \prime}$ :

$$
\lim _{\delta \rightarrow 0} \frac{1}{2 \pi i} \int_{\kappa_{\delta}\left(\lambda_{k}\right)} \frac{M(\mu)}{\lambda-\mu} d \mu=\frac{\alpha_{k}}{\lambda-\lambda_{k}} .
$$

By virtue of (4.2),

$$
\frac{1}{2 \pi i} \int_{\Gamma_{\delta}} \frac{M(\mu)}{\lambda-\mu} d \mu=\int_{\Omega_{\delta}} \frac{V(\mu)}{\lambda-\mu} d \mu .
$$

Hence, from (4.9) as $\delta \rightarrow 0$, we arrive at (4.7). Theorem 4.1 is proved.

In this section we study the following inverse problem

Inverse problem 2. Given the spectral data $S$, construct $\mathcal{L}$.

Let us formulate the uniqueness theorem for this inverse problem.

Theorem 4.2. If $S=\tilde{S}$, then $\mathcal{L}=\tilde{\mathcal{L}}$, i.e. $q(x)=\tilde{q}(x)$ a.e., $a=\tilde{a}, \beta=\tilde{\beta}, h=\tilde{H}, a_{1}=\tilde{a}_{1}$, $a_{2}=\tilde{a}_{2}$. Thus, the specification of the spectral data $S$ uniquely determines $\mathcal{L}$.

Theorem 4.2 follows from Theorems 3.2 and 4.1. Indeed, let $S=\tilde{S}$. By virtue of Theorem 4.1 , this yields $M(\lambda) \equiv \tilde{M}(\lambda)$. Using Theorem 3.2 we conclude that $\mathcal{L}=\tilde{\mathcal{L}}$.

Let us now give a constructive procedure for the solution of the inverse problem. For brevity, let $a_{2}$ be known. In order to construct the solution of the inverse problem we will use ideas of the method of spectral mappings [6]. The central role for solving the inverse problem will be played by the so-called main equation of the inverse problem which connects the spectral characteristics with the corresponding solutions of the differential equation. We give a derivation of the main 
equation, which is a linear equation in a suitable Banach space. It is important that the nonlinear inverse problem is reduced to the solution of the linear main equation. We prove the unique solvability of the main equation. Using the solution of the main equation we can construct all parameters of $\mathcal{L}$.

Let the spectral data $S$ be given. Using (4.7) we construct the Weyl function $M(\lambda)$. Then we calculate the number $\beta$ via (3.6), and the numbers $a, a_{1}$ using (2.12). Furthermore, we choose a model boundary value problem $\tilde{\mathcal{L}}$ such that $\tilde{a}=a, \tilde{\beta}=\beta, \tilde{a}_{1}=a_{1}$, and arbitrary in the rest (for example, one can take $\tilde{q}(x) \equiv 0$ and $\tilde{h}=0)$. Let $\tilde{S}=\left(\{\tilde{V}(\lambda)\}_{\lambda>0},\left\{\tilde{\lambda}_{k}, \tilde{\alpha}_{k}\right\}_{k \in \tilde{\omega}}\right), \tilde{\lambda}_{k}=\tilde{\rho}_{k}^{2}$ be the spectral data of $\tilde{\mathcal{L}}$. Denote $\lambda_{k 0}=\lambda_{k}, \lambda_{k 1}=\tilde{\lambda}_{k}, \alpha_{k 0}=\alpha_{k}, \alpha_{k 1}=\tilde{\alpha}_{k}$,

$$
\begin{gathered}
R(x, \lambda, \mu):=\frac{\langle\varphi(x, \lambda), \varphi(x, \mu)\rangle}{\lambda-\mu}=\int_{0}^{x} r(t) \varphi(t, \lambda) \varphi(t, \mu) d t \\
Q_{\lambda, \mu}(x)=R(x, \lambda, \mu) \hat{V}(\mu), Q_{n i, \mu}(x)=R\left(x, \lambda_{n i}, \mu\right) \hat{V}(\mu), \mu>0 \\
Q_{\lambda, k j}(x)=R\left(x, \lambda, \lambda_{k j}\right) \alpha_{k j}, Q_{n i, k j}(x)=R\left(x, \lambda_{n i}, \lambda_{k j}\right) \alpha_{k j}, \varphi_{k j}(x)=\varphi\left(x, \lambda_{k j}\right) .
\end{gathered}
$$

Similarly we define $\tilde{R}, \tilde{Q}_{\lambda, \mu}, \tilde{Q}_{\lambda, k j}, \tilde{Q}_{n i, \mu}, \tilde{Q}_{n i, k j}, \tilde{\varphi}_{k j}$ but with $\tilde{\varphi}$ instead of $\varphi$. If $\omega_{0} \neq \tilde{\omega}_{0}$, then we define the corresponding functions identically zero (for example, if $n \in \omega_{0} \backslash \tilde{\omega}_{0}$, then $\varphi_{n 1}=$ $\left.Q_{n 1, \mu}=Q_{n 1, k j}=Q_{\lambda, n 1}=0\right)$. Denote $\xi_{k}=\left|\rho_{k}-\tilde{\rho}_{k}\right|+\left|\alpha_{k}-\tilde{\alpha}_{k}\right|, \chi_{k}=1 / \xi_{k}$ if $\xi_{k} \neq 0$, and $\chi_{k}=0$ if $\xi_{k}=0$. Let $\omega^{\prime}:=\omega \cup \tilde{\omega}$, and let $\omega_{1}$ be the set of indices $v=(k, j)$, where $k \in \omega^{\prime}, j=0,1$. For $x<a, \lambda=\rho^{2}, \mu=\theta^{2}, \rho>0, \theta>0, n, k \in \omega^{\prime}, i, j=0,1$, we put

$$
\begin{gathered}
\psi_{\lambda}(x)=\varphi(x, \lambda) \exp (-\beta \rho x), \psi_{k 0}(x)=\left(\varphi_{k 0}(x)-\varphi_{k 1}(x)\right) \chi_{k}, \psi_{k 1}(x)=\varphi_{k 1}(x), \\
D_{\lambda, \mu}(x)=Q_{\lambda, \mu}(x) \exp (\beta(\theta-\rho) x), D_{\lambda, k 0}(x)=Q_{\lambda, k 0}(x) \exp (-\beta \rho x) \xi_{k}, \\
D_{\lambda, k 1}(x)=\left(Q_{\lambda, k 0}(x)-Q_{\lambda, k 1}(x)\right) \exp (-\beta \rho x), \\
D_{n 0, \mu}(x)=\left(Q_{n 0, \mu}(x)-Q_{n 1, \mu}(x)\right) \chi_{n} \exp (\beta \theta x), D_{n 1, \mu}(x)=Q_{n 1, \mu}(x) \exp (\beta \theta x), \\
D_{n 0, k 0}(x)=\left(Q_{n 0, k 0}(x)-Q_{n 1, k 0}(x)\right) \chi_{n} \xi_{k}, D_{n 1, k 0}(x)=Q_{n 1, k 0}(x) \xi_{k}, \\
D_{n 1, k 1}(x)=\left(Q_{n 1, k 0}(x)-Q_{n 1, k 1}(x)\right), \\
D_{n 0, k 1}(x)=\left(Q_{n 0, k 0}(x)-Q_{n 1, k 0}(x)-Q_{n 0, k 1}(x)+Q_{n 1, k 1}(x)\right) \chi_{n} .
\end{gathered}
$$

Similarly we define $\tilde{\psi}_{\lambda}, \tilde{\psi}_{k j}, \tilde{D}_{\lambda, \mu}, \tilde{D}_{\lambda, k j}, \tilde{D}_{n i, \mu}, \tilde{D}_{n i, k j}$. It follows from (2.6)-(2.7), (2.9), (2.12), (4.4)-(4.5) and Schwarz's lemma that for $x<a, \lambda=\rho^{2}, \mu=\theta^{2}, \rho>0, \theta>0, n, k \in$ $\omega^{\prime}, i, j=0,1$, the following estimates hold for large $\rho, \theta, n, k$ :

$$
\xi_{k}=O\left(k^{-1}\right), \quad \psi_{k j}(x)=O(1), \quad \frac{\partial^{j}}{\partial \lambda^{j}} \psi_{\lambda}(x)=O(1),
$$




$$
\begin{aligned}
D_{\lambda, \mu}(x), D_{n i, \mu}(x) & =O\left(\frac{\exp (-2 \beta \theta(a-x))}{\theta^{2}}\right), \\
D_{\lambda, k j}(x) & =O\left(\frac{\xi_{k}}{k}\right), \\
D_{n i, k j}(x) & =O\left(\frac{\xi_{k}}{(|n-k|+1)}\right) .
\end{aligned}
$$

The same estimates are valid for $\tilde{\psi}_{\lambda}, \tilde{\psi}_{k j}, \tilde{D}_{\lambda, \mu}, \tilde{D}_{n i, \mu}, \tilde{D}_{\lambda, k j}, \tilde{D}_{n i, k j}$.

Theorem 4.3. For each fixed $x<a$, the following relations hold:

$$
\left.\begin{array}{c}
\tilde{\psi}_{\lambda}(x)=\psi_{\lambda}(x)+\int_{0}^{\infty} \tilde{D}_{\lambda, \mu}(x) \psi_{\mu}(x) d \mu+ \\
\sum_{(k, j) \in \omega_{1}} \tilde{D}_{\lambda, k j}(x) \psi_{k j}(x), \lambda=\rho^{2}, \rho>0, \\
\tilde{\psi}_{n i}(x)=\psi_{n i}(x)+\int_{0}^{\infty} \tilde{D}_{n i, \mu}(x) \psi_{\mu}(x) d \mu+ \\
\sum_{(k, j) \in \omega_{1}} \tilde{D}_{n i, k j}(x) \psi_{k j}(x),(n, i) \in \omega_{1} .
\end{array}\right\}
$$

Proof. Take $\delta_{0}>0$ such that $\left|R e \rho_{k}\right|<\delta_{0},\left|R e \tilde{\rho}_{k}\right|<\delta_{0}$ for all $\rho_{k}^{2} \in B, \tilde{\rho}_{k}^{2} \in \tilde{B}$. Let $\gamma=\gamma_{+} \cup \gamma_{-}$ be the contour in the $\lambda$ - plane, where $\gamma_{+}:=\left\{\lambda=u+i v: v= \pm 2 \delta_{0}^{2}, u \geq 0\right\}$ and $\gamma_{-}:=\{\lambda=$ $\left.u+i v: u=\delta_{0}^{2}-v^{2} /\left(4 \delta_{0}^{2}\right), u \leq 0\right\}$ is the image of the set $P:=\left\{\rho=\sigma+i \tau: \sigma= \pm \delta_{0}, \tau \geq \delta_{0}\right\}$ under the map $\rho \rightarrow \lambda$. Denote by $\gamma_{N}:=\left(\gamma \cap\right.$ int $\left.R_{N}\right) \cup\left(R_{N} \cap\right.$ int $\left.\gamma\right)$ the closed contour (with counterclockwise circuit), and denote by $\gamma_{N}^{0}:=\left(\gamma \cap\right.$ int $\left.R_{N}\right) \cup\left(R_{N} \backslash\right.$ int $\left.\gamma\right)$ the closed contour (with clockwise circuit). Let

$$
\Omega_{\delta}^{\prime}:=B_{+} \backslash\left(\left(\bigcup_{\lambda_{k} \in B^{\prime \prime}}\left[\lambda_{k}-\delta, \lambda_{k}+\delta\right]\right) \bigcup\left(\bigcup_{\tilde{\lambda}_{k} \in \tilde{B}^{\prime \prime}}\left[\tilde{\lambda}_{k}-\delta, \tilde{\lambda}_{k}+\delta\right]\right)\right) .
$$

Consider the integral

$$
\varepsilon_{N, k}(x, \lambda):=\frac{1}{2 \pi i} \int_{R_{N}} \frac{T_{k}(x, \mu)-\delta_{1 k}}{\lambda-\mu} d \mu,
$$

where $\delta_{1 k}$ is the Kronecker delta, and $T_{k}(x, \lambda)$ are defined by (3.7). By virtue of (3.10),

$$
\lim _{N \rightarrow \infty} \varepsilon_{N, k}(x, \lambda)=0 .
$$

By Cauchy's integral formula,

$$
T_{k}(x, \lambda)-\delta_{1 k}=\frac{1}{2 \pi i} \int_{\gamma_{N}^{0}} \frac{T_{k}(x, \mu)-\delta_{1 k}}{\lambda-\mu} d \mu, \quad \lambda \in \operatorname{int} \gamma_{N}^{0},
$$


and consequently,

$$
T_{k}(x, \lambda)-\delta_{1 k}=\frac{1}{2 \pi i} \int_{\gamma_{N}} \frac{T_{k}(x, \mu)}{\lambda-\mu} d \mu-\varepsilon_{N, k}(x, \lambda) .
$$

Substituting into (3.8) we obtain

$$
\varphi(x, \lambda)=\tilde{\varphi}(x, \lambda)+\frac{1}{2 \pi i} \int_{\gamma_{N}} \frac{\tilde{\varphi}(x, \lambda) T_{1}(x, \mu)+\tilde{\varphi}^{\prime}(x, \lambda) T_{2}(x, \mu)}{\lambda-\mu} d \mu-\varepsilon_{N}(x, \lambda),
$$

where $\varepsilon_{N}(x, \lambda)=\tilde{\varphi}(x, \lambda) \varepsilon_{N, 1}(x, \lambda)+\tilde{\varphi}^{\prime}(x, \lambda) \varepsilon_{N, 2}(x, \lambda)$. By virtue of (4.13),

$$
\lim _{N \rightarrow \infty} \varepsilon_{N}(x, \lambda)=0
$$

Taking (3.7) into account we infer

$$
\begin{gathered}
\varphi(x, \lambda)=\tilde{\varphi}(x, \lambda)+\frac{1}{2 \pi i} \int_{\gamma_{N}}\left(\tilde { \varphi } ( x , \lambda ) \left(\varphi\left(x, \mu \tilde{\Phi}^{\prime}(x, \mu)-\Phi(x, \mu) \tilde{\varphi}{ }^{\prime}(x, \mu)\right)\right.\right. \\
+\tilde{\varphi}^{\prime}(x, \lambda)(\Phi(x, \mu) \tilde{\varphi}(x, \mu)-\varphi(x, \mu) \tilde{\Phi}(x, \mu)) \frac{d \mu}{\lambda-\mu}-\varepsilon_{N}(x, \lambda) .
\end{gathered}
$$

By virtue of (3.3), this yields

$$
\tilde{\varphi}(x, \lambda)=\varphi(x, \lambda)+\frac{1}{2 \pi i} \int_{\gamma_{N}} \tilde{R}(x, \lambda, \mu) \hat{M}(\mu) \varphi(x, \mu) d \mu+\varepsilon_{N}(x, \lambda),
$$

since the terms with $S(x, \mu)$ vanish by Cauchy's theorem.

Let $\Pi_{\delta}^{\prime}$ be the two-sided cut $\Pi_{0}$ without the $\delta$ - neighbourhoods of the points of $B^{\prime \prime} \cup \tilde{B}^{\prime \prime}$, and let

$$
\Gamma_{\delta}^{\prime}:=\Pi_{\delta}^{\prime} \bigcup\left(\bigcup_{\lambda_{k} \in B^{\prime \prime}} \kappa_{\delta}\left(\lambda_{k}\right)\right) \bigcup\left(\bigcup_{\tilde{\lambda}_{k} \in \tilde{B}^{\prime \prime}} \kappa_{\delta}\left(\tilde{\lambda}_{k}\right)\right)
$$

be the contour with counterclockwise circuit. Denote $\Gamma_{\delta, N}^{\prime}:=\Gamma_{\delta}^{\prime} \cap$ int $R_{N}$. Contracting the contour $\gamma_{N}$ in (4.15) to the real line and using the residue theorem, we calculate

$$
\begin{gathered}
\tilde{\varphi}(x, \lambda)=\varphi(x, \lambda)+\frac{1}{2 \pi i} \int_{\Gamma_{\delta, N}^{\prime}} \tilde{R}(x, \lambda, \mu) \hat{M}(\mu) \varphi(x, \mu) d \mu \\
+\sum_{\lambda_{k} \in B^{\prime},\left|\lambda_{k}\right|<r_{N}} \tilde{R}\left(x, \lambda, \lambda_{k 0}\right) \alpha_{k 0} \varphi_{k 0}(x)-\sum_{\tilde{\lambda}_{k} \in \tilde{B}^{\prime},\left|\tilde{\lambda}_{k}\right|<r_{N}} \tilde{R}\left(x, \lambda, \lambda_{k 1}\right) \alpha_{k 1} \varphi_{k 1}(x)+\varepsilon_{N}(x, \lambda) .
\end{gathered}
$$

Together with (4.14) this yields as $N \rightarrow \infty$ :

$$
\tilde{\varphi}(x, \lambda)=\varphi(x, \lambda)+\frac{1}{2 \pi i} \int_{\Gamma_{\delta}^{\prime}} \tilde{R}(x, \lambda, \mu) \hat{M}(\mu) \varphi(x, \mu) d \mu+
$$




$$
\sum_{k \in \omega^{0} \cup \tilde{\omega}^{0}}\left(\tilde{Q}_{\lambda, k 0}(x) \varphi_{k 0}(x)-\tilde{Q}_{\lambda, k 1}(x) \varphi_{k 1}(x)\right)
$$

Since

$\lim _{\delta \rightarrow 0} \frac{1}{2 \pi i} \int_{\kappa_{\delta}\left(\lambda_{k i}\right)} \tilde{R}(x, \lambda, \mu) \hat{M}(\mu) \varphi(x, \mu) d \mu=(-1)^{i} \tilde{R}\left(x, \lambda, \lambda_{k i}\right) \alpha_{k i} \varphi_{k i}(x), \lambda_{k 0} \in B^{\prime \prime}, \lambda_{k 1} \in \tilde{B}^{\prime \prime}$,

$\lim _{\delta \rightarrow 0} \int_{\Pi_{\delta}^{\prime}} \tilde{R}(x, \lambda, \mu) \hat{M}(\mu) \varphi(x, \mu) d \mu=\lim _{\delta \rightarrow 0} \int_{\Omega_{\delta}^{\prime}} \tilde{Q}_{\lambda, \mu}(x) \varphi(x, \mu) d \mu=\int_{0}^{\infty} \tilde{Q}_{\lambda, \mu}(x) \varphi(x, \mu) d \mu$,

it follows from (4.16) that for each $x<a, \lambda \in \mathbf{C}$ :

$$
\begin{aligned}
\tilde{\varphi}(x, \lambda)=\varphi(x, \lambda)+ & \int_{0}^{\infty} \tilde{Q}_{\lambda, \mu}(x) \varphi(x, \mu) d \mu+ \\
& \sum_{k \in \omega^{\prime}}\left(\tilde{Q}_{\lambda, k 0}(x) \varphi_{k 0}(x)-\tilde{Q}_{\lambda, k 1}(x) \varphi_{k 1}(x)\right),
\end{aligned}
$$

where the integral is understood in the principal value sense: $\int_{0}^{\infty}:=\lim _{\delta \rightarrow 0} \int_{\Omega_{\delta}^{\prime}}$. Taking our notations into account we conclude that (4.12) follows from (4.17). Theorem 4.3 is proved.

One can prove similarly that

$$
\begin{aligned}
D_{\lambda, \mu}(x)-\tilde{D}_{\lambda, \mu}(x) & +\int_{0}^{\infty} \tilde{D}_{\lambda, \xi}(x) D_{\xi, \mu}(x) d \xi+ \\
& +\sum_{(s, l) \in \omega_{1}} \tilde{D}_{\lambda, s l}(x) D_{s l, \mu}(x)=0, \lambda, \mu \in s(\mathcal{L}) \cup s(\tilde{\mathcal{L}}) .
\end{aligned}
$$

Let $m$ be the Banach space of bounded sequences $\alpha=\left[\alpha_{v}\right]_{v \in \omega_{1}}$ with the norm $\|\alpha\|_{m}=$ $\sup _{v \in \omega_{1}}\left|\alpha_{v}\right|$. Consider the vectors

$$
\psi(x)=\left[\psi_{v}(x)\right]_{v \in \omega_{1}}=\left[\begin{array}{c}
\psi_{k 0}(x) \\
\psi_{k 1}(x)
\end{array}\right]_{k \in \omega^{\prime}}, \tilde{\psi}(x)=\left[\tilde{\psi}_{v}(x)\right]_{v \in \omega_{1}}=\left[\begin{array}{c}
\tilde{\psi}_{k 0}(x) \\
\tilde{\psi}_{k 1}(x)
\end{array}\right]_{k \in \omega^{\prime}} .
$$

It follows from (4.10) that $\psi(x), \tilde{\psi}(x) \in m$ for each $x<a$. Let $G:=C_{1}[0, \infty)$ be the Banach space of continuously differentiable functions $f(\lambda)$ on the half-line $\lambda \geq 0$, such that $f(\lambda)$ and $\frac{\partial}{\partial \lambda} f(\lambda)$ are bounded, with the norm $\|f\|_{G}=\max _{j=0,1} \sup _{\lambda \geq 0}\left|\frac{\partial^{j}}{\partial \lambda^{j}} f(\lambda)\right|$. It follows from (4.10) that $\psi_{\lambda}(x), \tilde{\psi}_{\lambda}(x) \in G$ for each $x<a$. Consider the Banach space $\mathcal{B}$ of vectors

$$
F=\left[\begin{array}{l}
f \\
\alpha
\end{array}\right], \quad f \in G, \alpha=\left[\alpha_{v}\right]_{v \in \omega_{1}} \in m,
$$

with the norm $\|F\|_{\mathcal{B}}=\max \left(\|f\|_{G},\|\alpha\|_{m}\right)$. Let

$$
\Psi(x)=\left[\begin{array}{c}
\psi_{\lambda}(x) \\
\psi(x)
\end{array}\right], \quad \tilde{\Psi}(x)=\left[\begin{array}{c}
\tilde{\psi}_{\lambda}(x) \\
\tilde{\psi}(x)
\end{array}\right],
$$


Clearly, $\Psi(x), \tilde{\Psi}(x) \in \mathcal{B}$ for each fixed $x<a$. For each fixed $x<a$ we define the operator $\tilde{D}(x)$ : $\mathcal{B} \rightarrow \mathcal{B}$ by the formulas

$$
\begin{gathered}
\tilde{F}=\tilde{D}(x) F, \quad F=\left[\begin{array}{c}
f \\
\alpha
\end{array}\right] \in \mathcal{B}, \quad \tilde{F}=\left[\begin{array}{c}
\tilde{f} \\
\tilde{\alpha}
\end{array}\right] \in \mathcal{B}, \\
\tilde{f}(\lambda)=\int_{0}^{\infty} \tilde{D}_{\lambda, \mu}(x) f(\mu) d \mu+\sum_{v \in \omega_{1}} \tilde{D}_{\lambda, v}(x) \alpha_{v}, \lambda, \mu \geq 0, \\
\tilde{\alpha}_{u}=\int_{0}^{\infty} \tilde{D}_{u, \mu}(x) f(\mu) d \mu+\sum_{v \in \omega_{1}} \tilde{D}_{u, v}(x) \alpha_{v}, u=(n, i), v=(k, j) ; n, k \in \omega^{\prime}, i, j=0,1 .
\end{gathered}
$$

Similarly we define the operator $D(x)$. Using (4.11), by the well-known arguments (see, e.g. [7]) one can prove that for each fixed $x<a$, the operators $D(x)$ and $\tilde{D}(x)$ are linear bounded operators acting from $\mathcal{B}$ to $\mathcal{B}$.

Theorem 4.4. For each $x<a$, the vector $\Psi(x) \in \mathcal{B}$ is the solution of the equation

$$
\tilde{\Psi}(x)=(I+\tilde{D}(x)) \Psi(x)
$$

in the Banach space $\mathcal{B}$ ( $I$ is the identity operator). The operator $I+\tilde{D}(x)$ has a bounded inverse operator, i.e. Eq. (4.19) is uniquely solvable.

Proof. Relation (4.19) is equivalent to (4.12). Similarly, (4.18) takes the form $D(x)-\tilde{D}(x)+$ $\tilde{D}(x) D(x)=0$ or

$$
(I+\tilde{D}(x))(I-D(x))=I .
$$

Interchanging places for $\mathcal{L}$ and $\tilde{\mathcal{L}}$, we obtain in the same way

$$
\Psi(x)=(I-D(x)) \tilde{\Psi}(x), \quad(I-D(x))(I+\tilde{D}(x))=I .
$$

Hence the operator $(I+\tilde{D}(x))^{-1}$ exists, and it is a linear bounded operator. Theorem 4.4 is proved.

Equation (4.19) is called the main equation of the inverse problem. Solving (4.19) we find the vector $\Psi(x)$, and consequently, calculate the function $\varphi(x, \lambda)$ for $x \in[0, a-0]$ and $\lambda \in s(\mathcal{L})$. Since $\varphi(x, \lambda)$ is the solution of Eq. (1.1) and satisfies (1.2), we can construct the potential $q(x)$ a.e. on $(0, a)$, and the coefficient $h$. Thus, the inverse problem has been solved for the interval $x \in(0, a)$. In order to construct the potential $q(x)$ for $x>a$, we can act in the following way. 
Since we have constructed $q(x)$ for $x<a$ and $h$, it follows that the solutions $\varphi(x, \lambda)$ and $S(x, \lambda)$ are known for all $x \in[0, a-0]$ and $\lambda \in \mathbf{C}$. Using the jump conditions (1.3) we find $\varphi^{(\nu)}(a+0, \lambda)$ and $S^{(\nu)}(a+0, \lambda), \nu=0,1$. Denote

$$
\Phi_{a}(x, \lambda):=\Phi(x, \lambda) / \Phi^{\prime}(a+0, \lambda), x \in(a+0, \infty), \quad M_{a}(\lambda)=\Phi_{a}(a+0, \lambda) .
$$

Taking (3.3) into account we calculate

$$
M_{a}(\lambda)=\frac{S(a+0, \lambda)+M(\lambda) \varphi(a+0, \lambda)}{S^{\prime}(a+0, \lambda)+M(\lambda) \varphi^{\prime}(a+0, \lambda)} .
$$

Thus, the function $M_{a}(\lambda)$ is known for all $\lambda \in \mathbf{C}$. The function $M_{a}(\lambda)$ is the Weyl function for the classical Sturm-Liouville operator $\ell y:=-y^{\prime \prime}+q(x) y$ on the interval $(a, \infty)$ with the boundary condition $y^{\prime}(a)=0$. It is known (see, e.g. [4, Ch.2]) that the specification of $M_{a}(\lambda)$ uniquely determines the potential $q(x)$ a.e. on $(a, \infty)$. Moreover, using the method of spectral mappings [6] we can construct the potential $q(x)$ a.e. on $(a, \infty)$. Thus, the solution of the inverse problem can be found by the following algorithm.

Algorithm 1. Let the spectral data $S$ be given.

1) Calculate $M(\lambda)$ via (4.7).

2) Find $\beta$ by (3.6).

3) Calculate $a$ and $a_{1}$ using (2.12).

4) Choose a model boundary value problem $\tilde{\mathcal{L}}$ such that $a=\tilde{a}, \beta=\tilde{\beta}, a_{1}=\tilde{a}_{1}$ and arbitrary in the rest (for example, one can take $\tilde{q}(x) \equiv 0$ and $h=0$ ).

5) Construct $\tilde{\Psi}(x)$ and $\tilde{D}(x)$ for $x \in(0, a)$.

6) Find $\Psi(x), x \in(0, a)$ by solving the main equation (4.19).

7) Calculate $\varphi(x, \lambda)$ for $x \in(0, a), \lambda \in s(\mathcal{L})$.

8) Construct $q(x)$ for $x \in(0, a)$ and $h$.

9) Find $\varphi(x, \lambda)$ and $S(x, \lambda)$ for all $x \in[0, a-0]$ and $\lambda \in \mathbf{C}$.

10) Calculate $\varphi^{(\nu)}(a+0, \lambda)$ and $S^{(\nu)}(a+0, \lambda), \nu=0,1$, using (1.3).

11) Find $M_{a}(\lambda)$ via (4.20).

12) Construct $q(x)$ for $x>a$ from the given $M_{a}(\lambda)$ by the method of spectral mappings [6].

The solution of Inverse problem 1 can be found by the following algorithm.

Algorithm 2. Let the Weyl function $M(\lambda)$ be given.

1) Calculate $V(\lambda), \lambda \geq 0$ via (4.2).

2) Find $\lambda_{k} \in B^{\prime}$ and $\alpha_{k}$ as poles and residues of $M(\lambda)$.

3) Calculate $\lambda_{k} \in B^{\prime \prime}$ and $\alpha_{k}$ using (4.6).

4) Find $\beta$ by (3.6).

5) Calculate $a$ and $a_{1}$ using (2.12).

6) Construct $q(x)$ and $h$ by steps 4-12 of Algorithm 1 . 
Acknowledgment. This work was supported in part by Grant 19-01-00102 of Russian Foundation for Basic Research.

\section{References}

[1] W. Wasow, Linear Turning Point Theory, Springer-Verlag, Berlin/New York, 1985.

[2] J. McHugh, An historical survey of ordinary linear differential equations with a large parameter and turning points, Arch. Hist. Exact. Sci., 7 (1970), 277-324.

[3] K. Daho and H. Langer, Sturm-Liouville operators with an indefinite weight functions, Proc. Roy. Soc. Edinburgh Sect. A, 78 (1977), 161-191.

[4] G. Freiling and V. Yurko, Inverse Sturm-Liouville Problems and Their Applications, NOVA Science Publishers, New York, 2001.

[5] G. Freiling G. and V. Yurko, Inverse spectral problems for differential equations on the half-line with turning points, Journal of Differential Equations , 154 no.2 (1999), 419-453.

[6] V. A. Yurko, Method of Spectral Mappings in the Inverse Problem Theory, Inverse and Illposed Problems Series, VSP, Utrecht, 2002.

[7] G. Freiling and V. A. Yurko, Inverse spectral problems for singular non-selfadjoint differential operators with discontinuities in an interior point, Inverse Problems, 18 no. 3 (2002), 757-773.

Yurko, Vjacheslav Department of Mathematics, Saratov State University, Astrakhanskaya 83, Saratov 410012, Russia

E-mail: yurkova@info.sgu.ru 